\title{
Les enjeux des bibliothèques musicales à l'ère des pratiques culturelles numériques
}

The Impact of Digital Technology on Music Libraries

Los desafíos que las bibliotecas musicales deben enfrentar en

la era de la cultura digital

\section{Aline Moncey}

Volume 53, numéro 3, juillet-septembre 2007

URI : https://id.erudit.org/iderudit/1030646ar

DOI : https://doi.org/10.7202/1030646ar

Aller au sommaire du numéro

Éditeur(s)

Association pour l'avancement des sciences et des techniques de la

documentation (ASTED)

ISSN

0315-2340 (imprimé)

2291-8949 (numérique)

Découvrir la revue

Citer cet article

Moncey, A. (2007). Les enjeux des bibliothèques musicales à l'ère des pratiques culturelles numériques. Documentation et bibliothèques, 53(3), 143-152.

https://doi.org/10.7202/1030646ar

\section{Résumé de l'article}

L'évolution rapide des supports de diffusion soulève la question de l'adaptation des bibliothèques aux pratiques culturelles actuelles, qui intègrent désormais la consultation de contenus textuels et audiovisuels dématérialisés en ignorant souvent le droit de la propriété intellectuelle. Comment, dans ce contexte, les bibliothèques peuvent-elles se démarquer d'une offre de contenus facilement accessibles sur le Web - notamment grâce à l'intermédiaire de systèmes de téléchargement gratuits - en respectant le droit d'auteur et en veillant à la fois à rester en adéquation avec la demande des usagers ?
Tous droits réservés ( $)$ Association pour l'avancement des sciences et des techniques de la documentation (ASTED), 2007
Ce document est protégé par la loi sur le droit d'auteur. L’utilisation des services d'Érudit (y compris la reproduction) est assujettie à sa politique d'utilisation que vous pouvez consulter en ligne.

https://apropos.erudit.org/fr/usagers/politique-dutilisation/ 


\title{
Les enjeux des bibliothèques musicales à l'ère des pratiques culturelles numériques*
}

\author{
ALINE MONCEY \\ École nationale supérieure des sciences de l'information et des bibliothèques \\ Villeurbanne (France) \\ aline.moncey@hotmail.fr
}

\begin{abstract}
RÉSUMÉ | ABSTRACTS | RESUMEN
L'évolution rapide des supports de diffusion soulève la question de l'adaptation des bibliothèques aux pratiques culturelles actuelles, qui intègrent désormais la consultation de contenus textuels et audiovisuels dématérialisés en ignorant souvent le droit de la propriété intellectuelle. Comment, dans ce contexte, les bibliothèques peuvent-elles se démarquer d'une offre de contenus facilement accessibles sur le Web - notamment grâce à l'intermédiaire de systèmes de téléchargement gratuits - en respectant le droit d'auteur et en veillant à la fois à rester en adéquation avec la demande des usagers?
\end{abstract}

\section{The Impact of Digital Technology on Music Libraries}

The development of new means of disseminating information begs the following question: how do libraries adapt to the new cultural practices, including the consultation of texts and audiovisual materials, and still comply with copyright considerations? In that context, how do libraries offer a service that is better than what is easily accessible on the Web, especially given the free access to downloading systems, and still respect the copyright law and meet user needs?

Los desafios que las bibliotecas musicales deben enfrentar en la era de la cultura digital

La rápida evolución de los medios de difusión plantea el interrogante de la adaptación de las bibliotecas a las prácticas culturales actuales, que integran, de ahora en más, la consulta de contenidos textuales y audiovisuales desmaterializados, ignorando a menudo el derecho de Propiedad Intelectual. En ese contexto, ¿cómo pueden las bibliotecas destacarse frente a una oferta de contenidos de fácil acceso en Internet (en particular gracias a la ayuda de los sistemas de descarga gratuita) sin dejar de respetar los derechos de autor $y$ procurando adecuarse siempre a la demanda de los usuarios?

\section{Introduction}

I es mutations socioculturelles liées à l'évolution des technologies de l'information de ces dix dernières années voient s'affronter, dans les pays occidentaux notamment, deux visions des pratiques culturelles : l'une en faveur d'une réglementation de la consultation des œuvres culturelles par la voie de systèmes de contrôle qui restreignent l'utilisation des supports et, de ce fait, isolent l'individu face à l'œuvre, l'autre en faveur d'une ouverture du droit pour le partage des œuvres audiovisuelles, dans un but de socialisation de la culture. L'évolution rapide des supports de consultation pose par ailleurs la question de la place des médiathèques à l'heure du numérique. Comment ces dernières peuvent-elles s'adapter à des pratiques telles que le téléchargement de musique, qui sont aujourd'hui déjà très ancrées dans les mentalités et même revendiquées comme faisant partie des droits individuels (ou droits subjectifs), mais qui restent cependant majoritairement « hors-la-loi » en attendant de mieux être réglementées? Quel rôle les bibliothèques publiques, notamment musicales, puisque ce sont sans doute les plus touchées par le bouleversement des pratiques numériques actuelles, ont-elles à jouer dans une société où les biens culturels sont désormais considérés comme des biens de consommation ? Comment peuvent-elles s'adapter à la demande de leurs usagers sans aller à l'encontre du droit de la propriété intellectuelle? Quelles sont leurs possibilités? Quelles sont leurs limites ? Quel est l'avenir du document sonore en bibliothèque face au développement des nouveaux modes d'accès à la musique?

\section{Les pratiques culturelles numériques actuelles}

Si le débat autour des «nouvelles » pratiques culturelles numériques est aujourd'hui aussi virulent, c'est sans doute parce qu'il soulève une opposition nette entre une vision cadenassée de la connaissance et une vision communautaire des savoirs et cultures libres. De plus, au-delà de la défense affichée du patrimoine intellectuel semblent s'inscrire des motivations le plus souvent d'ordre financier, puisque de nouveaux modèles économiques se dessinent avec le développement de la
Texte produit dans le cadre du cours sur les documents visuels et sonore donné par M. James Turner à l'occasion d'un échange universitaire avec l'EBSI au semestre d'automne 2007-2008. 


\section{La pratique du téléchargement de musique, plutôt récente, peut-elle devenir une pratique culturelle reconnue et intégrée aux canaux classiques de la culture institutionnalisée?}

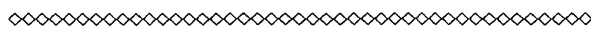

" société de l'information ". L'avènement de la diffusion gratuite des savoirs représente donc un manque à gagner pour les majors et autres industries culturelles. La question est donc de savoir comment faire cohabiter une culture libre, en profond et rapide changement, avec une conception plus contrôlée, pour que tous, auteurs, internautes, juristes, éditeurs et bibliothécaires, puissent trouver un équilibre juste.

\section{Les nouveaux outils de diffusion des contenus}

L'ouverture progressive des catalogues de bibliothèques à des supports tels que les $\mathrm{CD}, \mathrm{CD}-\mathrm{ROM}$ et aux logiciels doit aujourd'hui faire face à la multiplication de nouveaux formats de diffusion de contenus culturels dématérialisés et à l'apparition de matériel technique offrant toujours plus de fonctionnalités. Ainsi, les pratiques culturelles de centaines de milliers de consommateurs de biens culturels, qu'ils soient textuels ou audiovisuels, passent désormais par les technologies de l'information et de la communication (TIC), grâce notamment à l'utilisation d'Internet. En effet, la simplicité d'usage de ce medium et la facilité à disposer d'un large choix de titres, surtout musicaux et cinématographiques, à partir d'un ordinateur possédant une connexion Internet, accélère la mutation des habitudes informationnelles chez les jeunes générations. Citons en particulier la propagation de fichiers musicaux sous format $\mathrm{MP}_{3}$, qui est aujourd'hui largement répandue et a d'ores et déjà rencontré son public.

Plusieurs processus d'écoute de musique existent. La plus commune est celle du téléchargement de fichiers, qui offre la possibilité à l'internaute de disposer à sa guise du contenu d'une grande variété d'œuvres, protégées ou non, pouvant être stockées dans sa bibliothèque virtuelle personnelle. Le streaming, en revanche, est un moyen technique permettant d'écouter de la musique en ligne sans toutefois pouvoir la copier. Cette technique est par ailleurs souvent utilisée par les sites Web d'éditeurs de musique, d'artistes ou de radios en ligne, afin de promouvoir leurs nouvelles productions. Elle constitue donc une préécoute, pouvant être considérée par les industries de biens culturels comme des teasers. Il s'agit de permettre à l'utilisateur de tester un produit avant de l'acheter, car le but final est bien, pour la plupart des diffuseurs de musique en ligne via le strea- ming, la vente de leurs produits et non pas leur mise à disposition gratuite.

Plusieurs dispositifs coexistent donc, certains s'insérant dans un cadre juridique strict, alors que les autres sont toujours considérés comme étant illégaux. De nombreuses plateformes ont en effet vu le jour depuis les cinq dernières années : des plateformes de téléchargement de fichiers musicaux, pouvant être légales ou illégales. Les premières proviennent en partie d'éditeurs commerciaux développant des technologies alliant contenu et contenant, comme c'est le cas par exemple pour Apple, qui a développé le contenant iPod, qui est compatible avec la plateforme de téléchargement payant iTunes à partir de laquelle le téléchargement de fichiers est payant. Les autres plateformes sont quant à elles souvent issues de mouvements d'internautes en faveur du partage libre de contenus numériques mettant en réseau des individus qui échangent de la musique de poste-à-poste. Notons toutefois qu'il existe une différence entre les notions de " diffusion libre » et de gratuité, puisque la diffusion libre peut parfaitement concerner le téléchargement " libre " d'un nombre illimité de titres après acquittement de frais fixés par une plateforme légale, montants qui seront ensuite redistribués aux auteurs des œuvres et à leurs distributeurs. Notons enfin qu'il se développe également des licences de libre diffusion, notion sur laquelle nous reviendrons ultérieurement.

\section{Le rôle de la culture est-il antagoniste ou compatible avec les pratiques de téléchargement d'œuvres audiovisuelles?}

La question du rôle de la culture est sans doute sans fin, dans la mesure où de nombreuses réponses sont admissibles et où les évolutions des pratiques suscitent toujours de nouveaux questionnements qui ne sont pas nécessairement prévisibles. Par ailleurs, peut-on, dans l'absolu, prétendre qu'il existe une seule définition de la culture? Une seule conception de ce qui est acceptable ou ne l'est pas au nom de la culture ? Peut-on affirmer que les pratiques culturelles légales puissent être plus "valables " que celles qui privilégient le partage gratuit des contenus, sous prétexte qu'elles sont contrôlées par des professionnels de l'édition (mais qui sont aussi souvent dirigées par des professionnels en marketing) et non pas par des utilisateurs lambda qui ne possèdent pas d'expertise éditoriale mais partagent " seulement» des intérêts pour les mêmes œuvres ? Faut-il prendre en considération la question de l'éthique ou de la morale au cœur de ce débat : Est-il «bon » d'accepter toutes les évolutions technologiques sans condition? En d'autres termes, la pratique du téléchargement de musique, plutôt récente, peut-elle devenir une pratique culturelle reconnue et intégrée aux canaux classiques de la culture institutionnalisée? 
Il semblerait que les réponses potentielles à ces questions, si tant est qu'elles existent, dépendent de points de vue culturels inhérents aux différentes sociétés qui y sont confrontées. En effet, il serait intéressant de comparer les visions de deux régions du monde occidental comme l'Europe et l'Amérique du Nord, qui $a$ priori n'ont pas la même conception de la culture ni le même attachement à la notion de patrimoine. Si l'Europe reste encore de manière globale, puisqu'il serait inexact d'ignorer les différences de visions et de mentalités au sein de l'ensemble des pays de l'Union européenne, plus élitiste dans son rapport à l'accès à la culture ou dans ses choix d'acquisition en bibliothèque - ce qui présente certains avantages, comme l'assurance de la qualité du contenu informationnel des collections développées - les États-Unis et le Canada semblent quant à eux avoir adopté une démarche de partage et de diffusion des savoirs davantage tournée vers la notion d'intérêt public. Notons par ailleurs que cette vision d'intérêt public, ou vision communautaire, s'étend depuis environ 30 ans aux pays d'Europe grâce, notamment, au développement des bibliothèques de lecture publique. L'accent est désormais mis sur le rôle social de la culture, qui est non seulement créateur d'intérêts pour l'art, mais aussi créateur de liens entre les individus. Dans le cas de la musique, citons Peter Szendy, musicologue à l'Université Paris X - Nanterre, qui affirme que la musique suppose une appropriation de l'œuvre par celui qui l'écoute et qui, pour pouvoir être appréciée, nécessite d'être partagée (Latrive, 2004). Cette conception de la culture "partagée pour être appréciée " peut ici nous permettre d'avancer le fait que, pour que l'attrait des domaines artistiques parvienne à toucher des noninitiés, qui sont aujourd'hui plus facilement " abordables " grâce à l'intérêt grandissant pour les TIC dans l'opinion publique, il est absolument nécessaire de composer avec les nouvelles technologies pour renforcer les missions des bibliothèques de promotion et de diffusion de la culture et, pour cela, d'intégrer l'ensemble des nouvelles pratiques liées à l'utilisation d'Internet. En cela, le rôle de la culture ne semble pas être antagoniste aux nouvelles pratiques numériques mais, au contraire, semblerait s'inscrire dans une démarche d'encouragement au partage du patrimoine, qui servirait davantage l'intérêt public que les intérêts économiques. Au fond, les missions des bibliothèques restent les mêmes, seuls les publics et les supports de diffusion changent.

\section{La notion de pirate (hacker) : vers une pratique banalisée du téléchargement illégal ?}

Les réseaux de diffusion poste-à-poste (peer-topeer) sont, on ne s'en étonne pas, très en vogue à l'heure actuelle, comme le montrent un grand nombre d'études qui précisent, en outre, que la France est une grande consommatrice de ces nouvelles pratiques numériques. En effet, selon une enquête de TNS Sofres et une étude

\section{Le rôle de la culture ne semble pas être antagoniste aux nouvelles pratiques numériques mais, au contraire, semblerait s'inscrire dans une démarche d'encouragement au partage du patrimoine, qui servirait davantage l'intérêt public que les intérêts économiques.}

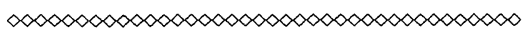

du ministère français de la Culture, le téléchargement est plébiscité par plusieurs générations d'internautes, notamment par les jeunes de 15 à 24 ans. Ainsi, " $17 \%$ des Français disent avoir téléchargé de la musique ou des films au cours des 12 derniers mois ", soit 1 français sur 6 , dont $14 \%$ disent avoir effectué leurs téléchargements gratuitement, $5 \%$ en payant, et $2 \%$ en cumulant les deux pratiques (Mercier, 2006). D’autres études révèlent de plus que le téléchargement illégal est très répandu, " [p] lus d'un internaute français sur deux pratique le téléchargement, [dont] $85 \%$ des fichiers sont téléchargés illégalement » et que, paradoxalement, le téléchargement légal est lui aussi en progression (Richard, 2007). Il semblerait donc que la pratique du téléchargement de contenu audiovisuel, notamment en dehors des canaux de diffusion régis par des éditeurs commerciaux, soit non seulement ancrée dans les mentalités, surtout chez les jeunes, mais aussi de plus en plus assumée. En d'autres termes, la génération pirate tant décriée par certains professionnels de l'industrie du disque et de l'industrie cinématographique, en s'étendant progressivement à toutes les couches de la société et en modifiant les modèles économiques en vigueur, prend peu à peu le pouvoir sur des modes de consommation payante de produits culturels aujourd'hui contestés, parce que trop restrictifs, et à but lucratif.

Remarquons à ce sujet que certaines données statistiques sur l'utilisation des plateformes de diffusion légales montrent que les systèmes payants occupent une part importante du marché. Une enquête de l'institut Piper Jaffray révèle en effet que : "Côté musique, c'est le règne d'Apple. Les baladeurs $M P_{3}$ de la marque iPod représentent $82 \%$ de part de marché parmi les possesseurs de baladeurs, contre $79 \%$ en 2006. De plus, $3 \%$ des personnes interrogées disent déjà posséder l'iPhone, et $9 \%$ projettent d'en acheter un dans les six prochains mois!» (Ratiatum.com, 2007.) Nous pouvons noter ici que la lutte commerciale entre les éditeurs de produits culturels s'intensifie et que la mise à disposition de musique en ligne n'est qu'un élément parmi la large gamme de produits qu'ils proposent. Un point important est toutefois soulevé : "Seule ombre au tableau, mais de taille, iTunes représente désormais $79 \%$ de part de marché pour l'achat de musique en ligne, contre $91 \%$ l'an dernier. " Ainsi pourrait-on douter de l'impact des 
Quant à la notion de "pirate», on peut rappeler qu'elle n'a pas toujours existé, même si la question du droit de la copie privée se posait déjà alors pour les emprunts en bibliothèque.

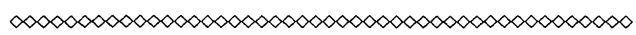

systèmes payants qui, à la suite de l'acquisition d'outils musicaux, leur sont couplés (comme c'est le cas ici avec l'iPod et son système de téléchargement iTunes, tous deux développés par Apple), puisqu'ils ne sont pas les seules sources d'approvisionnement des utilisateurs. Cependant, le choix de développer des outils d'écoute strictement verrouillés en dehors de l'utilisation de la plateforme à laquelle ils sont couplés apparaitrait totalement vain, puisqu'il éloignerait les acheteurs potentiels qui, à juste titre, pourraient se sentir captifs d'une pratique culturelle tournée vers la recherche de profits et qui est sans aucun doute contraire à la leur. Citons enfin une dernière interrogation très pertinente abordée dans la littérature foisonnante sur la question du téléchargement à l'heure actuelle : "Les plates-formes légales de téléchargement de musique vont-elles constituer de vrais relais de croissance pour l'industrie du disque?" (Devaux, 2007.)

Quant à la notion de "pirate", on peut rappeler qu'elle n'a pas toujours existé, même si la question du droit de la copie privée se posait déjà alors pour les emprunts en bibliothèque. Comme le précise Arsène Ott, président de l'Association de coopération des professionnels de l'information musicale (ACIM) : « $\grave{A}$ l'époque, on n'était pas considéré comme "pirates" et une redevance sur les supports enregistrables était supposée compenser les pertes des maisons de disques " (Ott, 2006). Pourquoi alors ce changement soudain de vision ? Pourquoi l'utilisation des supports numériques effraie-t-elle plus que toute autre évolution technique dans le passé ? Est-ce réellement la mise en danger des droits d'auteurs qui constitue la crainte majeure, ou est-ce plutôt la perte de profits pour l'industrie audiovisuelle?

\section{Les problèmes de droits d'auteur liés au partage des contenus}

La notion d'œuvre étant "bousculée " par les nouvelles pratiques culturelles, qui confèrent moins de pouvoir à l'auteur qu'au contenu intellectuel de sa création, il est important de retracer les évolutions du droit de la propriété intellectuelle pour comprendre les enjeux et les difficultés de mise en application des droits d'auteur à l'heure actuelle. Notons ici que les conceptions du droit d'auteur sont très variables d'un pays à l'autre et relèvent souvent de l'environnement culturel d'un pays et des limites de la notion de patrimoine qui y existent. On peut dégager deux tendances assez générales, l’une francophone, accordant plus de crédit à l'auteur et à son œuvre, et l'autre, anglophone, favorisant davantage les diffuseurs et exploitants de l'œuvre (BiblioDoc.Francophonie.org, 2007). Ces deux visions étant antagonistes, il apparait évident que la mutation des pratiques culturelles n'ait pas le même impact aujourd'hui selon les régions du monde.

\section{Le créateur au centre du processus de création : le droit de la propriété intellectuelle en France vs la loi sur le copyright aux États-Unis}

Selon Florent Latrive, auteur de $D u$ bon usage de la piraterie, "la propriété intellectuelle telle qu'elle fonctionne aujourd'hui [en France] ne reconnait pas la dimension collective de toute création. [...] La propriété intellectuelle sanctifie l'auteur ou l'inventeur, lui attribuant une place toute particulière dans une chaîne pourtant complexe et collective de création et d'originalité " (Latrive, 2004). Le droit confère donc à l'auteur un contrôle absolu sur sa création et sur les modalités d'utilisation de celle-ci, et ce, depuis la fin du XviII ${ }^{e}$ siècle, moment où les droits patrimoniaux de reproduction et de représentation ont été créés pour protéger les œuvres et leurs auteurs. C'est dans le respect de ce principe qu'est apparue la notion de copie privée, qui est une exception au droit d'auteur. En France, le droit de copie privée est toléré et est hors du contrôle de l'auteur, puisque, selon la loi, "les copies ou reproductions strictement réservées à l'usage privé $d u$ copiste et non destinés à une utilisation collective » (Latrive, 2004) sont autorisées, à la condition qu’elles soient réservées au cercle familial. On sanctionne donc la mise à disposition de l'œuvre, mais pas la copie proprement dite, même si elle est faite à partir d'un document emprunté en bibliothèque. Cette constatation a mené à la réglementation du droit de prêt en bibliothèque, en 2003, difficilement mis en place et toujours objet de débat aujourd'hui. Cette disposition garantit une rémunération des auteurs pour le manque à gagner encouru par la présence de leurs œuvres dans les collections de prêt des bibliothèques et qui, de ce fait, constitue autant de non-achats que de nombre d'emprunts, les usagers pouvant disposer du contenu d'œuvres de l'esprit qu'ils n'ont pas acquises via les circuits commerciaux d'édition, mais gratuitement ou presque grâce à leur abonnement en bibliothèque.

Aux États-Unis, en revanche, on parle de Fair use, exception au droit d'auteur ajoutée à la loi sur le copyright en 1976, qui reconnaît la critique, le commentaire, l'information journalistique, l'enseignement, les études universitaires, ainsi que la recherche comme étant légales et ne portant pas atteinte aux droits d'auteur (Wikipédia), tout comme le permet l'« utilisation équitable » au Canada. À travers ce modèle, ce n'est pas sur l'auteur que l'accent est mis, mais sur l'intérêt public, en encourageant les auteurs à produire des ouvres dans l'intérêt de la science. Dans une étude du Comité fran- 
çais IFLA portant sur les limites du droit d'auteur dans l'environnement numérique, il est précisé que «le droit d'auteur, [au départ réservé à l'auteur dans sa totalité], est souvent vendu ou attribué ensuite, totalement ou en partie, à un éditeur commercial, à un producteur de film, $\dot{a}$ un studio d'enregistrement ou à tout autre tiers qui exploitera l'œuvre commercialement. C'est pourquoi le droit d'auteur répond bien souvent davantage à des intérêts commerciaux qu'à ceux d'auteurs individuels " (Cfifla, 2006).

\section{Le débat entre généralisation des DRM (Digital Rights Management) et adoption de la licence globale : difficultés d'adoption d'une loi et incertitudes des politiques culturelles}

Pour tenter d'amender la loi sur les droits d'auteurs dans le contexte numérique que nous connaissons actuellement, le cadre juridique français a mis en vigueur la loi DADVSI, « Droits d'auteur et droits voisins dans la société de l'information ", élargissant les contours de la loi désormais applicable aux nouveaux supports, physiques ou dématérialisées, et visant à rendre obligatoires des mesures techniques capables de verrouiller l'utilisation frauduleuse de ces supports par tout individu. Ces mécanismes de verrouillage sont communément appelés DRM (Digital Rights Management) ou GND (Gestion numérique des droits). Ils visent à empêcher la copie d'une œuvre, son extraction vers un fichier numérique, ou la consultation d'un support sur une plateforme ou du matériel issus d'une compagnie concurrente. En cela, les DRM sont un frein à l'interopérabilité. Cette restriction d'usage des supports et des contenus, puisque ces derniers sont désormais tracés, peut toutefois soulever un problème éthique dans la mesure où elle constitue une atteinte au droit de copie privée, qui est, rappelonsle, une exception au droit d'auteur applicable en toute légalité au sein du cercle de famille. Ces systèmes de contrôle sont donc attaqués par de nombreux défenseurs de la diffusion et de l'accès libre aux cuvres parce que, en plus d'être liberticides, ils semblent davantage jouer en faveur d'intérêts économiques, comme nous l'avons vu plus haut, plutôt qu'en faveur des intérêts des auteurs. La loi DADVSI a donc suscité, et suscite toujours, de vives réactions de la part des professionnels de l'information comme des usagers.

Par ailleurs, on peut noter que le rejet de la loi DADVSI a conduit à l'émergence d'un autre modèle : celui de la "licence globale " qui, bien que faisant partie du projet originel d'amendement à la loi sur le droit d'auteur, n'a finalement pas été retenu. Ce modèle promouvait la mise en place d'une redevance ou rétribution forfaitaire aux ayants droit pour l'échange et le partage de contenus audiovisuels, et aurait dû permettre, à terme, de rémunérer les artistes plutôt que les distributeurs qui, actuellement, touchent une marge bénéficiaire importante sur les œuvres des auteurs qu'ils

\section{La situation juridique demeure donc floue dans la mesure où la loi DADVSI reste en vigueur seulement dans l'attente d'un compromis qui puisse satisfaire à la fois les usagers et les ayants droit.

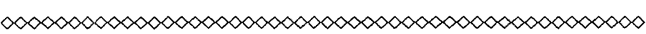

"signent ». Ainsi, l'accent aurait été mis sur la protection de l'œuvre elle-même et non pas sur la protection de son support, qui perd de son sens dans le contexte numérique que nous connaissons désormais. Malgré l'engouement de certains bibliothécaires pour la licence globale en France, ce modèle a été abandonné au profit de l'application de la loi DADVSI, aujourd'hui de plus en plus reconnue comme étant inadaptée tant d'un point de vue technique, parce que les systèmes de copy control peuvent endommager les supports de diffusion, qu'au point de vue éthique, parce qu'il est illusoire de penser qu'il est possible de contrôler tous les téléchargements de données textuelles et audiovisuelles grâce à des systèmes capables de tracer les "pirates ". La situation juridique demeure donc floue dans la mesure où la loi DADVSI reste en vigueur seulement dans l'attente d'un compromis qui puisse satisfaire à la fois les usagers et les ayants droit.

\section{La notion de connaissance ouverte ouvre la voie à un assouplissement de la notion d'œuvre, désormais autorisée au partage par leurs auteurs, dans le respect du droit de la propriété intellectuelle}

Le mouvement open source, dont sont issus les logiciels libres qui connaissent un succès certain au sein des communautés d'internautes, a récemment donné naissance à une licence de libre diffusion grâce à laquelle les ayants droit peuvent définir des degrés d'utilisation de leurs œuvres et ainsi permettre leur libre circulation auprès du public qui prendra le relais et pourra diffuser ces contenus en toute légalité, y compris sur les réseaux poste-à-poste. La Licence Creative Commons s'organise autour d'un contrat de droit que l'auteur signe afin de délimiter les usages qu'il autorisera sur son œuvre selon quatre critères, comme il est stipulé sur le site Web français de Creative Commons :

Paternité : L’œuvre peut être librement utilisée, à la condition de l'attribuer à son auteur en citant son nom.

Pas d'utilisation commerciale : Le titulaire de droits peut autoriser tous les types d'utilisation ou, au contraire les restreindre aux utilisations non commerciales (les utilisations commerciales restant soumises à son autorisation). 
Le rôle des bibliothèques est de mettre en valeur le patrimoine culturel d'un État donné et du reste du monde, afin d'offrir à ses publics une représentation exhaustive des savoirs et des cultures.

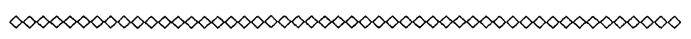

Pas de modification : Le titulaire de droits peut continuer à réserver la faculté de réaliser des œuvres dérivées ou au contraire autoriser à l'avance les modifications, traductions...

Partage à l'identique des conditions initiales : À la possibilité d'autoriser à l'avance les modifications peut se superposer l'obligation, pour les œuvres dites dérivées, qu'elles soient proposées au public avec les mêmes libertés (sous les mêmes options Creative Commons) que l'œuvre originaire (Creative commons France).

Ainsi, le but est de permettre la libre circulation d'œuvres textuelles, audiovisuelles ou photographiques, dans le respect de l'intégrité de leurs auteurs. Notons que cette démarche est à but non lucratif et "contribu[e] à diminuer les coûts de trainsaction: distribution, recherche automatique d'œuvres, information sur le régime des droits, négociation en cas d'utilisation [...], rédaction d'autorisations d'utilisation ou de remix, [etc.] " (Creative commons France). Cette nouvelle approche est par ailleurs en vogue dans les communautés scientifiques, où les représentants de la culture savante mettent de plus en plus en avant la co-création ainsi que la promotion d'un modèle de culture et de savoirs libres pour tous et loin des préoccupations marchandes des industries culturelles. Ici encore, on retrouve l'idée de circulation de l'information à des fins d'intérêt public et d'éveil à la culture, artistique ou scientifique. Ce n'est donc plus la notion de support qui prime, mais celle d'accès libre aux contenus.

Cela pose donc une nouvelle fois la question de l'adaptation des nouvelles pratiques au sein des bibliothèques qui, par définition, ont pour mandat premier de stocker et de mettre en valeur des contenus enregistrés sur des supports de diffusion.

\section{L'évolution des bibliothèques : vers l'ouverture des supports de diffusion?}

L'ouverture des catalogues à de nouveaux supports, amorcée depuis une vingtaine d'années, se poursuit aujourd'hui avec l'ajout de documents numériques qui offrent des contenus sans support physique mais virtuel. Notons ici que la notion de "dématérialisation » peut être discutable, dans la mesure où les contenus se doivent d'être stockés sur un support tel qu'un serveur informatique pour être consultables. Les bibliothèques tentent en tout cas, de cette manière, de garantir une adéquation entre les besoins des usagers et les évolutions des technologies. Cependant, on peut se poser la question de savoir quelles sont les limites à la promotion de la modernité des bibliothèques, qui pourraient, d'une part, perdre en crédibilité si elles intégraient trop vite les nouvelles pratiques culturelles sans les avoir intégralement analysées et, d'autre part, risquer de se trouver rapidement en perte de vitesse face à leurs usagers si elles n'étaient pas suffisamment réactives aux innovations de la société de l'information. Les difficultés d'adaptation sont donc majeures, notamment en raison du contexte actuel de surcharge d'informations, qui ne présentent pas toujours nécessairement de valeur informationnelle forte.

\section{Rôles et missions de l'institution " bibliothèque publique »}

Les bibliothèques publiques ont pour missions traditionnelles de mettre en valeur et de disséminer l'ensemble des cultures, de promouvoir les auteurs et artistes locaux, nationaux et internationaux, de garantir la gratuité de l'accès aux documents pour tous et de sélectionner des contenus au sein d'une masse documentaire toujours plus importante dans le contexte numérique. Elles doivent faire le pont entre la culture populaire et la culture savante, notions qui ne sont pas sans rapport avec les orientations politiques d'un pays. Yves Alix, rédacteur en chef du Bulletin des bibliothèques de France (BBF), démontre que la dimension politique entre en ligne de compte dans le rôle d'éducation des bibliothèques ainsi : "l'enjeu essentiel [est aussi] de garantir à tous les citoyens l'accès aux sources d'informations et à la documentation, et de contribuer à l'action culturelle démocratique " (Alix, 200o : 24). L'accès à l'information et la diversité des sources doivent donc être démocratiques pour que l'institution "bibliothèque publique " prenne tout son sens. Antigone Mouchtouris, sociologue spécialisée dans le domaine de la culture, précise aussi que "l'œuvre est légitimée par les institutions, mais puise sa vraie force et sa valorisation dans la réception que le public lui fait " (Mouchtouris, 2003 : 35). Ainsi, le rôle des bibliothèques est-il de mettre en valeur le patrimoine culturel d'un État donné et du reste du monde, afin d'offrir à ses publics une représentation exhaustive des savoirs et des cultures. À l'inverse, le rôle du public est, lui, de légitimer la présence des œuvres acquises par les bibliothèques en allant les consulter. Toutefois, il serait illusoire de penser que les politiques culturelles aient pour but de former, voire d'éduquer les usagers (Mouchtouris, 2003), mais qu'elles occupent plutôt une place de "médiateur » en donnant à voir à un public peu initié certains fonds plus difficiles d'accès que d'autres.

La notion de médiation est par ailleurs très en vogue actuellement, plus particulièrement la notion de médiation numérique. Ce processus consiste en effet, pour les professionnels de l'information, à sélectionner 
des contenus numériques dans la masse documentaire en constante augmentation, la difficulté de la tâche résidant dans le fait que cette sélection doive s'effectuer à l'intention d'un public de plus en plus " expert " en recherche d'information, dans la mesure où ce dernier n'a pas d'autre choix que de trier lui-même de l'information pertinente parmi la quantité de documentation qui le submerge chaque jour sur son ordinateur personnel. L'une des questions qui émergent alors est la suivante : Quelle est la valeur ajoutée des bibliothèques dans ce contexte de surabondance de l'information?

S’il est vrai que les usagers sont appelés à être autonomes de plus en plus jeunes face à leurs besoins documentaires, l'apport des professionnels de l'information ne serait pas négligeable, selon Silvère Mercier, bibliothécaire et auteur du blogue Bibliobsession 2.0, dans un billet intitulé "La médiation numérique dans les bibliothèques, une voie d'avenir " (Mercier, 2007a). En effet, deux notions majeures y apparaissent : la « qualification des ressources » et la «valorisation des compétences ». Il s'agit de guider les usagers et de leur permettre de trouver des documents pertinents à travers un vaste choix documentaire au moyen de recommandations (recommandations statistiques, professionnelles, et commentaires d'utilisateurs). Ainsi, le travail des bibliothécaires est mis en valeur par un processus d'orientation du lecteur, qui pourra alors naviguer dans le catalogue entre titres connus (qu'il aura lui-même cherchés) et inconnus (que les bibliothécaires auront sélectionnés et qui seront suggérés automatiquement selon les requêtes exprimées). Notons que de nombreuses bibliothèques publiques et universitaires ont désormais développé leurs propres services de questions-réponses et de repérage de documents selon des thématiques clairement identifiées, qui constituent des points de repère fiables pour les usagers et correspondent à la mission de diffusion des bibliothèques. Cela répond également à la logique de la "longue traîne ", qui a pour but de faire éclater la concentration du marché culturel en redirigeant les utilisateurs vers des documents de qualité qui n'ont pas bénéficié d'effets de réseau capables de leur assurer une notoriété suffisante pour être visibles.

\section{Réflexions sur la mort annoncée des bibliothèques musicales : Faut-il y croire?}

L'avenir des bibliothèques musicales suscite aujourd'hui beaucoup d'inquiétude chez les discothécaires, étant donné la tendance à la baisse du nombre d'emprunts de CD et la popularité toujours grandissante du téléchargement chez une part importante des consommateurs de biens culturels, pour certains habitués des bibliothèques (Saglio, 2007). D'où l'émergence de nombreux questionnements : Les usages d'Internet et de ses ressources audiovisuelles remettent-ils en cause l'utilité des bibliothèque musicales? Comment les bibliothèques peuvent-elles se démarquer d'une offre de

\section{Est-il raisonnable d'envisager que le public puisse, dans un avenir proche, se détourner de manière suffisamment importante des services musicaux proposés par les bibliothèques pour entraîner leur disparition?}

$\infty<\infty<\infty<\infty<\infty<\infty<\infty<\infty<\infty<\infty<\infty<\infty<\infty<\infty<\infty<\infty<\infty$

contenu accessible sur le Web ? La mort annoncée du $\mathrm{CD}$ peut-elle entraîner la mort des bibliothèques musicales ? Mais aussi : Le fait d'intégrer progressivement de nouveaux contenus audiovisuels dématérialisés peut-il ralentir leur perte de vitesse ? Ou encore : Les pratiques culturelles sur le Web qui, selon certains points de vue, isolent l'internaute peuvent-elles modifier les comportements au point de faire chuter la fréquentation des bibliothèques et de se passer de leur rôle social?

S'il n'existe pas de réponse univoque, on peut néanmoins noter que les nombreux débats qui ont cours actuellement autour de ces questions sont une preuve de la bonne santé du milieu bibliothéconomique et de la remise en question, positive et nécessaire, des bibliothèques par les bibliothécaires eux-mêmes. Malgré l'absence de certitudes, certains professionnels n'en annoncent pas moins la mort du CD d'ici environ cinq ans. Ils conseillent tout de même de continuer à enrichir les collections et à expérimenter de nouveaux services, tout en repensant la notion de " collection de prêt ", qui disparaît dans le contexte numérique (Bruthiaux, 2007). Si l'inquiétude est par ailleurs légitime quant à l'avenir des collections audio traditionnelles, comment ne pas se demander si lesdits bibliothécaires inquiets ne sont, en réalité, pas trop alarmistes ? En effet, est-il raisonnable d'envisager que le public puisse, dans un avenir proche, se détourner de manière suffisamment importante des services musicaux proposés par les bibliothèques pour entraîner leur disparition ? Peut-on réellement croire que le tout-numérique puisse l'emporter sur des collections traditionnelles auxquelles les usagers sont attachés ? Ces derniers pourraient-ils en effet se détacher de l'objet physique, du support de consultation lui-même? S'il est indéniable qu'une grande partie des jeunes générations privilégient désormais l'écoute et la découverte de nouveautés par voie électronique, il semble difficile de penser que ce mode de consommation culturelle puisse réellement se transmettre à l'ensemble des générations, étant donné la valeur affective accordée aux supports audio - notamment au CD, pour la majeure partie de la population. Cette valeur affective, par ailleurs, ne saurait être transférée de manière équivalente dans un contexte numérique, puisque s'il est vrai que le contenu est l'essence même d'un document, on ne peut pas ignorer que le rôle que joue un support dans l'appréciation d'une œuvre par des auditeurs est grand, dans la mesure où le support et ce qui l'entoure apportent une valeur ajoutée 


\section{La sérendipité peut certes s'organiser selon les goûts des usagers, mais elle reste plus efficace lorsqu'elle est complétée par la médiation de professionnels de l'information.}

$\infty 00000000000000000000000000000000000000$

au document sonore (comme la présence d'informations contenues dans le livret d'un CD ou le travail graphique réalisé). Notons qu'il est possible d'intégrer une partie de ces "métadonnées " sur une interface Web, comme une plateforme de téléchargement, mais celles-ci resteront somme toute virtuelles!

Il est également difficile de concevoir que l'ensemble des usagers puissent totalement se passer du contact humain que peut leur procurer les bibliothèques, qui est une composante majeure de leur fréquentation. L'aspect social que l'on met aujourd'hui volontiers en avant, comme nous l'avons vu plus haut, est en effet indissociable des compétences des bibliothécaires en matière de conseil. Or s'il est désormais possible de faire des découvertes sans médiation humaine, grâce à des systèmes informatiques capables d'exploiter la longue traîne en redirigeant les internautes vers des œuvres qu'ils n'étaient pas venus chercher en premier lieu, cette capacité de conseil n'est en rien comparable à celle que peuvent offrir des bibliothécaires musicaux spécialistes de leurs domaines, puisque dans leur mission même est inscrite la promotion de la rareté, qui est le contre-pied à l'effet de concentration que peuvent entraîner les logiques de recommandation des usagers sur le Web (parce que, du fait du nombre toujours croissant d'internautes utilisant les sites de partage, les redirections vers des artistes moins connus auront plus de chances d'alimenter à leur tour la culture de masse...). En d'autres termes, la sérendipité peut certes s'organiser selon les goûts des usagers, mais elle reste plus efficace lorsqu'elle est complétée par la médiation de professionnels de l'information, qui sont aptes à proposer des choix «nouveaux » et « authentiques », d'où la nécessité de faire cohabiter services traditionnels et innovations techniques.

\section{Solutions alternatives déjà en place ou en projet dans les bibliothèques}

À ce jour, de nombreuses bibliothèques ont déjà tenté de développer des services intégrant les nouvelles pratiques culturelles, à savoir la consultation de contenus virtuels diffusés de manière légale, étant donné que la protection du droit d'auteur est primordiale en bibliothèque. On peut mentionner la mise en place de «burn stations» qui permettent aux usagers de graver des fichiers musicaux libres de droit sur $\mathrm{CD}$, ou l'installation de bornes USB permettant de télécharger de la musique en ligne directement sur un lecteur $\mathrm{MP}_{3}$. On peut aussi évoquer l'affiliation de certaines bibliothèques à des plateformes de téléchargement payant comme le propose le service iThèque, qui est un service de prêt numérique de mise à disposition de fichiers musicaux, audiovisuels et textuels (e-books) protégés par des systèmes de contrôle (DRM) et qui sont, de ce fait, chronodégradables après la période de prêt autorisée, ainsi que protégés contre toute copie ou altération du contenu. Si l'on peut comprendre la tentation des bibliothécaires de développer des systèmes capables d'offrir à leurs usagers un fonds de titres dématérialisés tout en respectant les droits d'auteur, force est de constater que cette offre est reconnue aujourd'hui comme étant mal adaptée aux besoins des utilisateurs. Plusieurs expériences le montrent, dont celle de la médiathèque de Martigues (France), "qui a ouvert un service d'écoute sur place de musique, accessible avec des PC-Pocket connectés par wifi sur le serveur de stockage. Il a fallu numériser des milliers de morceaux de musique. L'expérience est présentée avec beaucoup de réserves tant sur la lourdeur du système que sur le succès relatif. " (Bruthiaux, 2007). En d'autres termes, l'envie d'adapter une offre numérique en fonction des nouvelles habitudes de consommation de produits culturels des usagers est certes défendable et très certainement à encourager, mais il semblerait que les difficultés d'ordre technique soient encore trop importantes pour pouvoir appliquer de telles offres sous leurs formes actuelles. N'est-il donc pas trop tôt pour prendre des décisions à long terme ? À ce propos, citons Yves Alix qui précise que, "à l'heure actuelle, l'absence d'offres fiables destinées aux bibliothèques laisse à penser qu'il est prématuré de proposer des offres de téléchargement de fichiers aux usagers, la filière musicale étant elle-même à la recherche de nouveaux modèles permettant notamment d'assurer un financement à la création. » (Mercier, 2007b)

Par ailleurs, se pose le problème de double emploi possible entre des titres musicaux présents à la fois dans les collections physiques et dans les fichiers téléchargeables. Comment y remédier ? Mais surtout est-il nécessaire d'y remédier? La question semble en effet se poser dans la mesure où, lorsque l'offre de contenus musicaux numériques sera stabilisée, les usagers qui emprunteront des supports de nature différente (traditionnels $v s$ numériques) ne seront pas nécessairement les mêmes. Laissera-t-on courir le risque à des usagers peu à l'aise avec les formats virtuels de ne pas pouvoir emprunter le titre qui les intéresse, si celui-ci se trouve uniquement sur support numérique ? Comment déciderons-nous alors du choix de titres à faire figurer dans les différentes collections? À quels publicss'adresseront-ils? Les critères d'acquisition de fichiers numériques seront-ils semblables aux critères d'acquisition de documents traditionnels ? En outre, la notion de collection est peut-être elle aussi à remettre en question, puisque certains documents, comme les périodiques électroniques, ne constituent désormais plus une source pérenne d'information 
dans le contexte numérique, dans la mesure où l'arrêt d'un abonnement entraîne souvent la perte de l'intégralité des données. En effet, les bibliothèques, qui investissent pourtant des sommes très importantes auprès des agences d'abonnement à des revues en ligne pendant une période moyenne de trois ans, n'acquièrent finalement qu'un " accès " à l'information et non à son contenu, l'arrêt d'un abonnement "effaçant " souvent les numéros précédemment " acquis». Des solutions alternatives se mettant progressivement en place pour éviter cette disparition des collections électroniques, peut-être pouvons-nous penser qu'il en sera de même pour le stockage des fichiers musicaux s'ils venaient à se généraliser dans les bibliothèques.

\section{Conclusion}

S'il n'existe pas encore de véritable solution répondant aux problèmes rencontrés actuellement par les bibliothécaires quant à la transposition des nouvelles pratiques numériques à l'échelle des bibliothèques, on n'en constate pas moins que le monde bibliothéconomique est bien présent et qu'il se positionne de manière générale en faveur de l'intégration des contenus virtuels. Si l'avenir du CD constitue une réelle inquiétude et risque, à long terme, de faire diminuer le nombre de discothécaires dans les services offrant de la musique à leur clientèle, on peut toutefois douter de la disparition totale de ce médium, jadis annoncé comme étant le support de conservation pérenne de l'information par excellence, qui, tout comme le disque vinyle aujourd'hui, continuera sans doute à rassembler les amateurs, attachés non pas uniquement à des contenus musicaux, mais aussi à leur support. Par comparaison, on pourrait mentionner les craintes ressenties dans le monde du théâtre lorsque le cinéma s'est fortement développé, ou lorsque le monde du cinéma lui-même a été inquiété par l'arrivée de la télévision dans les années 1950. Il semblerait que chaque évolution technique se pose comme concurrente directe du média précédemment prisé par le public. Cependant, ces craintes sont-elles réellement fondées?

Les bibliothèques, en profonde mutation à l'heure actuelle, doivent donc redéfinir leur rôle dans le contexte numérique afin de faire la démonstration de leur « utilité sociale ". Le compromis pourrait, comme nous l'avons vu plus haut, se situer autour de l'adoption d'un modèle de licence de libre diffusion, dans un souci de respecter la propriété intellectuelle et de promouvoir la culture savante et de divertissement pour tous. Certains bibliothécaires musicaux parlent en effet du retour possible de la licence globale, afin de garantir la diversité créative qui est, rappelons-le, l'une des missions premières des bibliothèques publiques. D'autres, comme Dana Hilliot, fondateur du label Another Record diffusant des artistes indépendants sous licence Creative Commons, ajoutent que la bibliothèque, par opposition aux plateformes d'écoute commerciales et à l'industrie du divertisse-

\section{Les bibliothèques, en profonde mutation à l'heure actuelle, doivent donc redéfinir leur rôle dans le contexte numérique afin de faire la démonstration de leur " utilité sociale».}

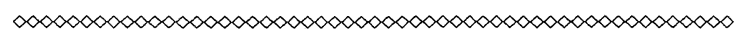

ment en général, offre "une pluralité de pratiques musicales, [...] des espaces pour le silence, et pour des événements sonores réalisés indépendamment des circuits de production et de diffusion gérés par le marché " (in Ott, 2006). Citons enfin Silvère Mercier, qui affirme très justement que l'intégration progressive de nouveaux services virtuels dans les bibliothèques "illustre [l']une des tendances à l'ouvre, celle de faire de la bibliothèque un lieu d'expérience musicale. À l'heure où les pratiques donnent une place très importante au "live" et à la mobilité, il est intéressant de repenser le rôle et les moyens de la bibliothèque comme lieu et source de l'écoute sur place" (Mercier, 2007b). Ainsi pourrait s'inscrire la valeur ajoutée des bibliothèques dans le contexte numérique, plus encore que dans le monde analogique : vers une médiation des contenus toujours plus nombreux réalisée par des bibliothécaires soucieux de la qualité et de la rareté d'artistes méconnus du grand public, faisant de la bibliothèque un espace d'expérience musicale et un relais entre le monde de la culture et les usagers désirant être guidés au sein de la culture de masse. L'avenir semble donc plus que jamais se trouver dans la médiation. (-)

\section{Sources consultées}

\section{Monographies}

Alix, Yves, et al. 200o. Le droit d'auteur et les bibliothèques, Paris, Éditions du Cercle de la Librairie, $237 \mathrm{p}$.

Latrive, Florent. 2004. Du bon usage de la piraterie. Culture libre, sciences ouvertes, Paris, Exils, $170 \mathrm{p}$.

Mouchtouris, Antigone. 2003. Sociologie du public dans le champ culturel et artistique, Paris, L'Harmattan, $130 \mathrm{p}$.

Sites Web et blogues

BiblioDoc.Francophonie.org. 2007. «Les droits d'auteurs», 7 octobre: <»»http://bibliodoc.francophonie.org/article.php3?id article $=121>$. (Consulté le 14 octobre 2007.)

Bruthiaux, Pierre. 2007. "Musique en ligne en bibliothèque publique ", Bulletin des bibliothèques de France, 2007, $\mathrm{n}^{\circ} 3$ : 105-106 : <mmhttp://bbf.enssib.fr/sdx/BBF/frontoffice/2007/o3/ document.xsp?id=bbf-2007-03-0105-006/2007/03/fam-tourhorizon/tourhorizon\&nDoc $=1 \&$ statutMaitre $=$ non\&statutFils $=$ no n\&tri $=>$. (Consulté le 14 octobre 2007.)

Cfifla.asso.fr. - CLM (Comité sur le copyright et autres problèmes juridiques) 2006. "Les limitations et les exceptions au droit d'auteur et aux droits voisins dans l'environnement numérique : le point de vue des bibliothécaires au niveau international ", Michèle Battisti (trad.), 29 août : <m»http://www.cfifla. 
asso.fr/accueilifla/exceptiond roitauteurcorps.htm>. (Consulté le 14 octobre 2007.)

Creative commons France. "Description du projet iCommons en France " : <mmhttp://fr.creativecommons.org/description.htm>. (Consulté le 14 octobre 2007.)

«Les options et les contrats disponibles": <m»http:// fr.creativecommons.org/contrats.htm>. (Consulté le 14 octobre 2007.)

Devaux, Guillaume. 2007. "Téléchargement légal : un marché en pleine maturation ", Le journal du Net, 30 avril : <mmhttp://www. journaldunet.com/ebusiness/commerce/dossier/300407-platesformes-telechargement-musique-itunes-virgin-fnac/index. shtml>. (Consulté le 14 octobre 2007.)

Mercier, Silvère. 2007a. «La médiation numérique dans les bibliothèques, une voie d'avenir ", Bibliobsession 2.0, 28 juin : <m»http:// bibliobsession.free.fr/dotclear/index.php?2007/06/28/257-lamediation-numerique-dans-les-bibliotheques-une-voie-davenir $>$. (Consulté le 14 octobre 2007.)

2007b. "La musique dématérialisée et les bibliothèques : Que se passe-t-il? Que fait-on? ", Bibliobsession 2.0, 26 juin : <m»http://bibliobsession.free.fr/dotclear/ index.php?2007/06/26/254-la-musique-dematerialisee-et-lesbibliotheques-que-se-passe-t-il-que-fait-on>. (Consulté le 14 octobre 2007.)

http :. 2006. " $\mathrm{P}_{2} \mathrm{P}$, musique, DRM et bibliothèques... Ithèque et consorts à l'affût ", Bibliobsession 2.0, 26 octobre : <m»http:// bibliobsession.free.fr/dotclear/index.php?2006/10/26/110-p2p- musique-drm-et-bibliothequesitheque-et-consorts-a-l-affut $>$. (Consulté le 14 octobre 2007.)

Ott, Arsène. 2006. " La musique a-t-elle encore sa place dans les médiathèques? ", ACIM : portail des bibliothécaires musicaux, 4 octobre : <mmhttp://www.acim.asso.fr/article.php3?id_ article $=175>$. (Consulté le 14 octobre 2007.)

Ratiatum.com. Tous vos loisirs numériques. 2007. «Chez les étudiants américains le piratage baisse, iTunes aussi ", 11 octobre : <mmhttp://www.ratiatum.com/breve5817_Chez_les_etudiants_ americains_le_piratage_baisse_iTunes_aussi.html $>$. (Consulté le 14 octobre 2007.)

Richard, Philippe. 2007. «Les Français toujours accros au téléchargement illégal ", oinet, 9 février : <m»http://www.oinet.com/ article/340983.html $>$. (Consulté le 14 octobre 2007.)

Saglio, Thomas. 2007. "Musique en bibliothèque : la fin des haricots? ", ACIM : portail des bibliothécaires musicaux, 24 août : <mmhttp://www.acim.asso.fr/spip.php?article202>. (Consulté le 14 octobre 2007.)

Wikipédia. "Fair use " : <»mhttp://fr.wikipedia.org/wiki/Fair_ use\#Port.C3.A9e_du_Copyright $>$. (Consulté le 14 octobre 2007.) 